\title{
Surgical correction for lower lid epiblepharon in Asians
}

Kyung In Woo, Kayoung Yi, Yoon-Duck Kim

\begin{abstract}
Backgroundlaims-Epiblepharon is a congenital lid anomaly in which a fold of skin and underlying orbicularis muscle push the lashes against the eyeball. It is important to get a good lash eversion effect without forming a prominent lid crease in Asian patients. The surgical effect of this rotating suture technique was evaluated.
\end{abstract}

Methods-Surgical correction for epiblepharon was performed on 197 patients and the results analysed in 169 patients who had been followed for 1 month or more. After subciliary incision, several buried 8-0 nylon sutures were placed to allow adhesion between the tarsal plate and the subcutaneous tissue of the upper skin flap with minimal resection of pretarsal orbicularis and redundant skin.

Results-156 patients $(92.3 \%)$ showed satisfactory results during 7.1 months of average follow up. Reoperation was performed only on two patients out of 13 because of mildness of symptoms and signs. Complications were minimal including suture abscesses in four patients and wound dehiscence in one.

Conclusion-The rotating suture technique was very effective in repairing epiblepharon without forming a prominent lower eyelid crease.

(Br f Ophthalmol 2000;84:1407-1410)

Department of

Ophthalmology,

Sungkyunkwan

University School of

Medicine, Kangbuk

Samsung Hospital,

Seoul, Korea

K I Woo

Samsung Medical

Center, Seoul, Korea

Y-D Kim

Department of

Ophthalmology,

National Medical

Center, Seoul, Korea

K Yi

Correspondence to: Dr Yoon-Duck Kim Department of

Ophthalmology, Samsung

Medical Center, 50,

Ilwon-dong, Kangnam-gu,

Seoul, Korea, 135-230

ydkim@smc.samsung.co.kr

Accepted for publication 16 June 2000
Epiblepharon is a congenital lid anomaly, in which a fold of skin and underlying orbicularis muscle tilt the lashes, often pushing them against the globe. It is one of the most common eyelid abnormalities among Asian children. ${ }^{1}$ Although some cases resolve with facial growth during childhood, ${ }^{12}$ epiblepharon may cause corneal injury and astigmatism from the lashes and skin fold. ${ }^{3}$ Surgical correction is needed for the cases in which there is significant corneal injury from the lashes.

The anatomical structure of the lower eyelid in Asian people has been reported to be different from that of white people. ${ }^{4}$ Besides the anatomical differences, the surgeon must also take into consideration that many Asian patients may have different beauty standards and cultural backgrounds. In western countries, it is common practice to correct epiblepharon by forming an adhesion between the anterior lamella of the eyelid and the lower eyelid retractors usually by making a lower eyelid crease..$^{5-7}$ Many Asians, however, do not have this lower eyelid crease. A prominent lower eyelid crease resulting from the surgery of epiblepharon should be avoided in Asians because most of them do not want it (Fig 1).

We describe a simple surgical technique to correct epiblepharon without making a lower eyelid crease. We reviewed the cases which had been operated for epiblepharon and evaluated the effect of our procedures.

\section{Patients and methods}

Surgery was performed on cases with significant corneal erosions and/or irritative symptoms. One hundred and ninety seven patients had been operated by one author (Y-D Kim) with our technique for epiblepharon, from January 1990 to December 1997.

Anaesthesia and haemostasis of the eyelid were obtained by injecting a mixture of $2 \%$ lignocaine (lidocaine), $0.5 \%$ bupivacaine with 1:100 000 adrenaline (epinephrine) for cooperative patients, and injecting a mixture of $1 \%$ lidocaine with 1:200000 adrenaline under general anaesthesia for uncooperative patients. The skin was incised along the infralash line, 1-2 $\mathrm{mm}$ below the most inferior line of the lashes, through the whole eyelid length or medial two thirds in accordance with the severity of epiblepharon. Dissection was carried out between the tarsal plate and skin-muscle flap exposing the tarsal plate. After applying thermal cauterisation to the bleeding points, the subcutaneous tissue of the upper skin flap was sutured to the exposed tarsal plate with 8-0 nylon interrupted sutures in a buried fashion to rotate the direction of the lashes. In severe cases needing more eversion, the suture was placed on the inferior end of the tarsal plate or on the capsulopalpebral fascia. Three to six sutures were placed. Small amounts of orbicularis muscle and redundant skin overlying the lower lid margin were removed. In the medial and lateral ends of the incision, the dogears were removed with triangular skin excision. After meticulous haemostasis, the skin was

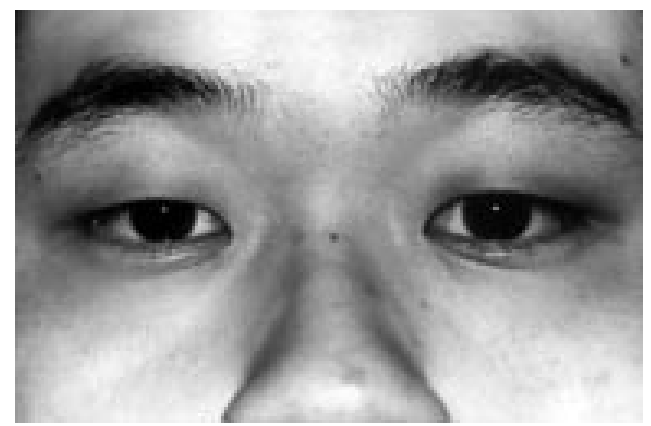

Figure 1 This patient required removal the lower eyelid crease which occurred as a result of epiblepharon surgery. $H e$ was displeased with his cosmetic result even though no cilia touched his cornea. 
closed with a running $6-0$ fast absorbing plain gut suture (Fig 2).

All the patients received ofloxacin eye ointment (Tarivid, Hoechst Marion Roussel) twice daily for 1-2 weeks until the suture was completely absorbed.

\section{Results}

We analysed the results of 169 patients who had been followed for 1 month or more. The patients ranged in age from 6 months to 32 years (median age 6 years). There were 96 males and 73 females. All were Korean and had epiblepharon involving the lower eyelid. Seven patients $(4.1 \%)$ had at least one previous
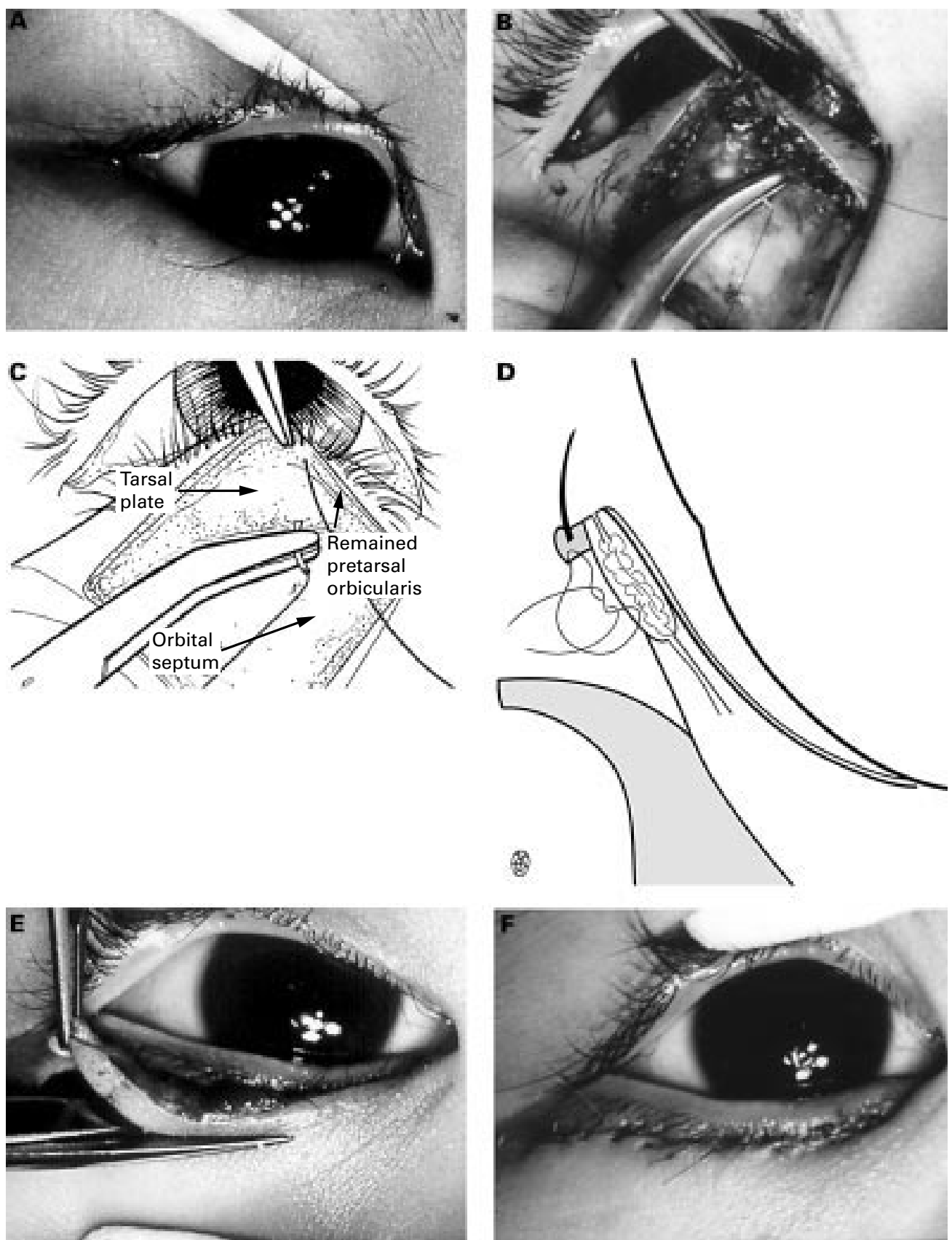

Figure 2 Surgical techniques. (A) Note the vertical direction of the eyelashes. (B, C) Buried 8-0 nylon sutures are placed to allow adhesions to form between the tarsal plate and the subcutaneous tissue of the upper skin flap. (D) Cross section demonstrates suture in its proper position. (E) Minimal amounts of redundant skin and orbicularis muscle are removed to avoid ectropion or lower eyelid retraction. In both ends of the incision, the dogears are removed with triangular skin excision. (F) After meticulous haemostasis, the skin is closed with a running 6-0 fast absorbing gut suture. 


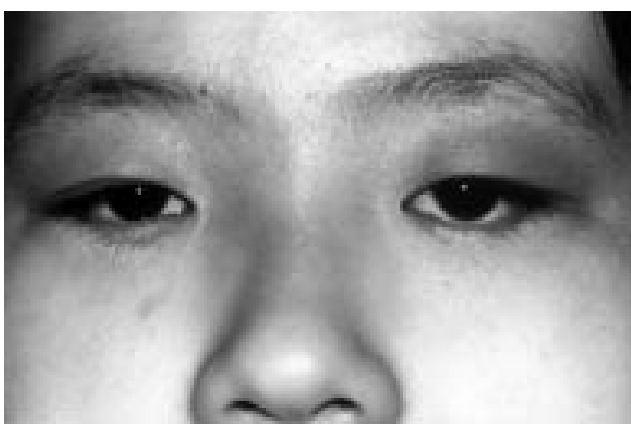

Figure 3 This patient had epiblepharon surgery 10 years ago, and she has visible scars and lower eyelid retraction because too much skin had been taken at the time of surgery.

7.1 months of follow up. There was no case of lid retraction or ectropion. A skin crease did not form with good cosmesis. There were 13 cases of recurrence, and time of recurrence was variable. Seven cases were found to recur after 4 months after surgery, three cases after 8 months, two cases after 12 months, and one case at 25 months after the surgery. Reoperations were performed only on two patients out of 13 because of mildness of symptoms and signs. These two patients underwent the same operation and recovered completely through the follow up period of 5 and 33 months respectively. Complications were minimal including suture abscesses in four patients and wound dehiscence in one.

\section{Discussion}

Epiblepharon is a fold of skin, which overlaps the eyelid margin and pushes the lashes against the cornea. ${ }^{8}$ It involves the medial one third or one half of the lower eyelid and usually occurs bilaterally. The cilia in the involved section of the eyelid show a vertical direction. The tarsal platform in epiblepharon does not roll inward but remains in its normal position. It should be differentiated from the extremely rare congenital entropion, which is characterised by an inward rotation of the entire tarsus and lid margin. ${ }^{9-11}$

The anatomy of the lower lid is analogous to that of the upper lid. The anatomical defect in epiblepharon is the absence of the adhesion between the lower eyelid retractors and the anterior lamella, allowing the skin and muscle to roll upwards..$^{5-9}$ This is similar to the anatomical difference of the upper lid in Asians with no eyelid crease. ${ }^{12}$ Like the upper lid, the main anatomical differences of the lower eyelid in Asians are higher septum attachment onto the tarsus or inferior retractor and the absence of retractor fibres into the skin and orbicularis. ${ }^{5}$ These anatomical differences explain the absence or poor lower eyelid crease in Asian patients and why epiblepharon is so common in Asian children.

Epiblepharon needs surgical treatment only when there are severe ocular irritating symptoms or significant keratitis, as it tends to disappear spontaneously with age. The object of surgical treatment is to make an adhesion between the anterior lamella of the eyelid and the lower eyelid retractors. Several surgical procedures such as full thickness eyelid sutures, ${ }^{13}{ }^{14}$ buried sutures, ${ }^{1} \mathrm{~V}-\mathrm{Y}$ plasty, ${ }^{215}$ and excision of the skin and orbicularis muscle ${ }^{816-19}$ have been used as treatment for epiblepharon.

Full thickness sutures are simple and fast, as it corrects the basic pathophysiological defects by creating a scar between the skin, orbicularis, and eyelid retractor, producing a cosmetically acceptable eyelid crease, and avoiding sacrificing the normal eyelid tissue. ${ }^{13}{ }^{14}$ This procedure, however, showed a high recurrence rate, up to $29 \%,{ }^{1}$ and is recommended only for mild cases.

Excision of the skin and orbicularis muscle with tarsal fixation (modified Hotz procedure, Hotz-Celsus procedure) has been the most commonly performed procedure. ${ }^{816-18}$ This procedure is simple and has been successful in correcting epiblepharon. However, lower eyelid retraction or ectropion could result from this procedure if too much skin or orbicularis is removed (Fig 3). In addition, recurrence of epiblepharon could also be a problem if too small an amount of skin is removed.

Some textbooks of oculoplastic surgery ${ }^{20} 21$ recommend correcting epiblepharon by forming an adhesion between the anterior lamella of the eyelid and the lower eyelid retractors usually by making a lower eyelid crease. However, most Asian patients do not want to have a lower eyelid crease for cosmetic reasons. Therefore, we always try not to make one.

Our rotating suture technique has several distinctions: (1) the skin incision is made just below the lash line instead of the lower margin of tarsus in order not to form a lower eyelid crease and visible scar. (2) The vertically oriented eyelashes are rotated by making an adhesion between the subcutaneous tissue of the upper skin flap and the tarsal plate with 3-6 buried rotating sutures. (3) The amount of rotation is adjusted by the placement of sutures onto the tarsus. When we need more rotatation, we place the sutures on the more inferior part of the tarsus. As a suture material, we prefer 8-0 nylon to absorbable sutures to lessen the recurrence rate. (4) Minimal amounts of the redundant skin and orbicularis muscle are removed to avoid ectropion or lower eyelid retraction.

There were 13 cases $(7.7 \%)$ of recurrence with our technique. Of the 13 recurrent patients, only two patients $(1.3 \%)$ had significant symptoms and needed a second operation. There was no case of lid retraction or ectropion. No patients were displeased with their cosmetic result. Other complications were minimal including suture abscesses in four and wound dehiscence in one patient. This rotating suture technique is simple and very effective in fixing lower eyelid epiblepharon and showed good cosmesis without having to make a lower eyelid crease.

1 Hayasaka S, Noda S, Setogawa T. Epiblepharon with inverted eyelashes in Japanese children. II. Surgical repairs. Brf Ophthalmol 1989;73:128-30.

2 Johnson CC. Epiblepharon. Am f Ophthalmol 1968;66: 1172-5.

3 Khwarg SI, Lee YJ. Epiblepharon of the lower eyelid: classification and association with astigmatism. Korean $f$ Ophthalmol 1997;11:111-7. 
4 Carter SR, Seiff SR, Grant PE, et al. The Asian lower eyelid: a comparative anatomic study using high-resolution magnetic resonance imaging. Ophthalmic Plast Reconst Surg 1998;14:227-34

5 Jordan R. The lower-lid retractors in congenital entropion and epiblepharon. Ophthalmic Surg 1993;24:494-6.

6 O'Donnell BA, Collin JRO. Congenital lower eyelid deformity with trichiasis (epiblepharon and entropion). Aust NZ F Ophthalmol 1994;22:33-7.

7 Millman AL, Mannor GE, Putterman AM. Lid crease and capsulopalpebral fascia repair in congenital entropion and epiblepharon. Ophthalmic Surg 1994;25:162-5.

8 Levitt JM. Epiblepharon and congenital entropion. $A m f$ Ophthalmol 1957;44:112-3.

9 Jordan DR. Lid crease and capsulopalpebral fascia repair. Ophthalmic Surg 1995;26:91.

10 Tse DT, Anderson RL, Fratkin JD. Aponeurosis disinsertion in congenital entropion. Arch Ophthalmol 1983;101:

11 Bartley GB, Nerad JA, Kersten RC, et al. Congenital entropion with intact lower eyelid retractor insertion. $\mathrm{Am} \mathcal{f} \mathrm{Oph}$ thalmol 1991;112:437-41.

12 Doxanas MT, Anderson RL. Oriental eyelids. An anatomic study. Arch Ophthalmol 1984;102:1232-5.
13 Quickert MH, Rathbun E. Suture repair of entropion. Arch Ophthalmol 1971;85:304-5.

14 Quickert MH, Wilkes TDI, Dryden RM. Nonincisional correction of epiblepharon and congenital entropion. Arch Ophthalmol 1983;101:778-81.

15 Johnson CC. Epicanthus and epiblepharon. Arch Ophthalmol 1978;96:1030-3.

6 Hotz FC. A new operation for entropion and trichiasis. Arch Ophthalmol 1979;8:249-63.

17 Hotz FC. Remarks on 177 operations for entropium and trichiasis. Arch Ophthalmol 1882;11:442-50.

18 Karlin DB. Congenital entropion, epiblepharon, and antimongoloid obliquity of the palpebral fissure. $A m \mathcal{F}$ Ophthalmol 1960;50:485-93.

19 Beyer-Machule CK. Lids. In: Heilmann K, Paton D, eds. Atlas of ophthalmic surgery. Techniques - complications. Vol 1. Stuttgart: Georg Thieme Verlag, 1985:2-3.

20 Rathbun JE. Entropion. In: Hornblass A, ed. Oculoplastic, orbital, and reconstructive surgery. Vol 1. Eyelids. Baltimore: Williams \& Wilkins, 1988:309-11.

21 Martin RT, Nunery WR, Tanenbaum M. Entropion, trichiasis, and distichiasis. In: McCord CD Jr, Tanenbaum M, Nunery WR, eds. Oculoplastic surgery. 3rd ed. New York: Raven Press, 1995:221-2.

\section{Contributors please note:}

Communications from all countries except the UK and Republic of Ireland should be sent to Professor C Hoyt, Editor, British fournal of Ophthalmology, University of California, Department of Ophthalmology, 10 Kirkham Street, K 301, San Francisco, CA 94143-0730, USA (tel: 001415 502-6871; fax: 001415 514-1521).

Manuscripts from the UK and the Republic of Ireland should be sent to Professor Andrew Dick, UK Editor, British fournal of Ophthalmology, Division of Ophthalmology, University of Bristol, Lower Maudlin Street, Bristol BS1 2LX (tel: +44 (0) 0117 929-4496; fax: +44 (0) 117 929-4607). 